\title{
Ytterbium Doped Nano-crystalline Optical Fiber for Reduced Photodarkening
}

\author{
S. Yoo, M. P. Kalita, A. J. Boyland, A. Webb, R. J. Standish, and J. K. Sahu \\ Optoelectronics Research Centre, University of Southampton, Southampton SO17 1BJ, United Kingdom \\ Email: sey@orc.soton.ac.uk \\ M. C. Paul, S . Das, S . K . Bhadra and M . Pal \\ Fiber Optics Laboratory, Central Glass and Ceramic Research Institute, CSIR, Kolkata 70032, India
}

\begin{abstract}
We report suppression of photodarkening in Yb-doped nano-crystalline fibers in silica host. The photodarkening induced loss reduced by 20 times compared to Yb-doped aluminosilicate fibers. The laser efficiency of the nano-crystalline fiber was $79 \%$.

2010 Optical Society of America

OCIS codes: (160.2290) Fiber materials; (060.2320) Fiber optics amplifiers and oscillators
\end{abstract}

\section{Introduction}

Ytterbium-doped silica fiber lasers has been a front runner in the race for higher powers due to its excellent conversion efficiency. However, pump induced photodarkening in Yb-doped fibers has been recognized as a bottleneck for power scaling in many applications. The photodarkening process is host material dependent. The induced loss is much more pronounced when the $\mathrm{Yb}^{3+}$ ions are incorporated in aluminosilicate host than in phosphosilicate host [1,2]. Thus, the photodarkening appears to be suppressed by modifying the host composition.

Here, we investigate $\mathrm{Yb}$ doped $\mathrm{Y}_{2} \mathrm{O}_{3}$ (or $\mathrm{Y}_{3} \mathrm{Al}_{6} \mathrm{O}_{12}$ ) nano-crystalline in a silica rich matrix for reduced photodarkening. $\mathrm{Y}_{2} \mathrm{O}_{3}$ is an attractive material for laser applications and efficient $\mathrm{Yb}^{3+}: \mathrm{Y}_{2} \mathrm{O}_{3}$ ceramic lasers have been reported in $[3,4]$. It is expected that $\mathrm{Y}_{2} \mathrm{O}_{3}$ nanoparticles will provide different surroundings for the $\mathrm{Yb}^{3+}$ ions which helps to reduce the photodarkening even in high $\mathrm{Yb}^{3+}$ concentration.

\section{Experiments and results}

The $\mathrm{Yb}$-doped $\mathrm{Y}_{2} \mathrm{O}_{3}$ (or $\mathrm{Y}_{3} \mathrm{Al}_{6} \mathrm{O}_{12}$ ) nanoparticles were attained from $\mathrm{SiO}_{2}-\mathrm{Al}_{2} \mathrm{O}_{3}-\mathrm{P}_{2} \mathrm{O}_{5}-\mathrm{Li}_{2} \mathrm{O}-\mathrm{BaO}$ core glass of an optical fiber preform through a standard MCVD-solution doping technique. The presence of crystalline nanoparticles in a heat treated preform were confirmed under transmission electron microscope (TEM) measurement [5]. The D-shaped fibers were pulled in double clad structure from the preforms and high power operation was demonstrated with good lasing efficiency [5]. Figure 1 represents refractive index profile of the nano-crystalline fibers with cross-sectional view in the inset. The core diameter was $18 \mathrm{~m}$ with 0.136 of core NA. The background loss was $\sim 70 \mathrm{~dB} / \mathrm{km}$ at $1285 \mathrm{~nm}$ measured by high resolution OTDR. The Yb concentration was $\sim 6000 \mathrm{ppm} \mathrm{wt} \%$.

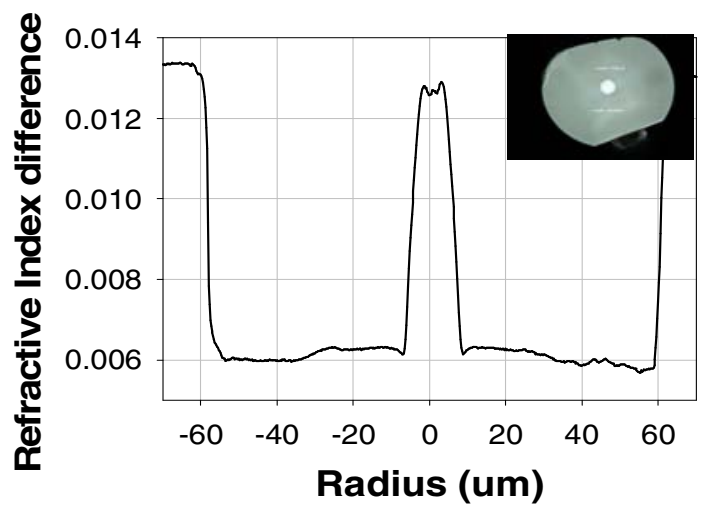

Fig. 1. Refractive index profile of $\mathrm{Yb}^{3+}$-doped nano-crystalline fiber. Inset: cross-sectional view of the fiber

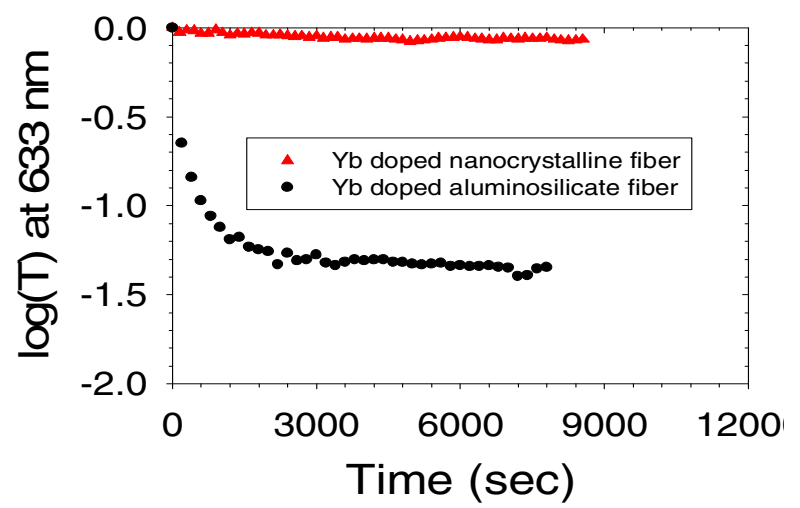

Fig. 2. Temporal characteristics of transmitted power at $633 \mathrm{~nm}$ for $\mathrm{Yb}$ doped nano-crystalline fiber and aluminosilicate fiber

The photodarkening of the Yb-doped nano-crystalline fibers was evaluated by monitoring the transmitted output power through the fiber under $965 \mathrm{~nm}$ irradiation. We used fiber-coupled single mode laser diode as a pump source. The output end of the pump fiber was spliced to the fiber under test. A He-Ne laser at $633 \mathrm{~nm}$ was used as a 
probe beam which coupled to the nano-crystalline fiber and propagated the same direction as the pump beam. The probe beam was chopped and the output was detected by photodiode and lock-in amplifier. We used $1 \mathrm{~cm}$ of the fibers to suppress the amplified spontaneous emission. We measured photodarkening induced loss of a Yb-doped aluminosilicate fiber fabricated in-house for comparison. The pump input power was maintained to provide $\sim 35 \%$ of population inversion of $\mathrm{Yb}^{3+}$. The temporal characteristics of the transmitted probe power are represented in Fig. 2. The photodarkening induced loss is significantly reduced in Yb-doped nano-crystalline fiber. When we fitted the measured results with stretched exponential form, we found that the saturated induced loss in Yb-doped nanocrystalline fiber reduced by 10-20 times compared to the aluminosilicate counterpart, depending on the host material compositions.

The laser efficiency of the fiber was tested under $976 \mathrm{~nm}$ end pumping configuration. A linear 4\%- 4\% reflecting cavity was formed by perpendicularly cleaved end facets. A dichroic mirror at the pump throughput end separated the pump and signal wavelength. The laser performance is shown in Fig. 3(a). The slope efficiency was $79 \%$ with respect to the launched pump power. Figure 3(b) shows the laser spectrum which is quite broad covering from 1036-1072 $\mathrm{nm}$. The measurement resolution was $1 \mathrm{~nm}$.
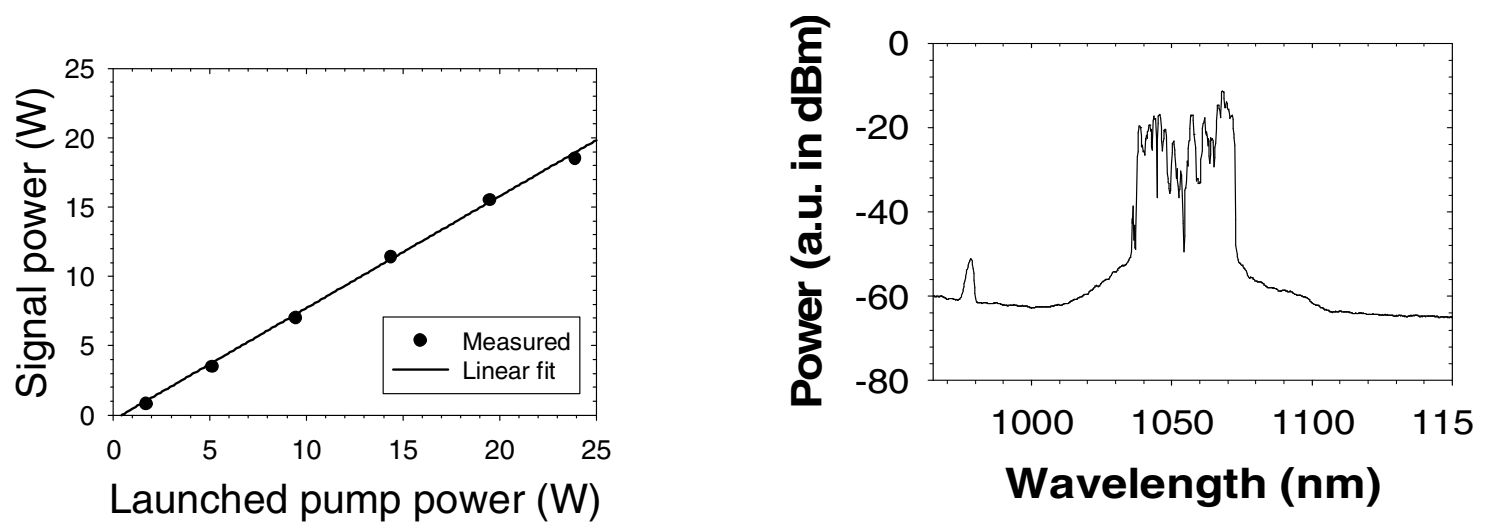

Fig. 3. Laser performance of Yb-doped nano-crystalline fiber (a) and laser operating wavelength (b)

\section{Conclusion}

In conclusion, we reported $\mathrm{Yb}$-doped $\mathrm{Y}_{2} \mathrm{O}_{3}$ nano-crystalline fibers fabricated by a conventional MCVD-solution doping technique. The fiber exhibited low photodarkening induced loss compared to the Yb-doped aluminosilicate fiber, with good laser efficiency. This class of fibers will keep the advantage of the mechanical properties of silica glass, whereas the surrounding of RE ions can be engineered by varying the nanoparticle compositions.

Acknowledgement: The authors would like to acknowledge CSIR and Royal Society,UK for provision of financial support under CSIR-Royal Society Joint Collaborative Research Work.

\section{References}

[1] S. Jetschke, S. Unger, A. Schwuchow, M. Leich, and J. Kirchhof, "Efficient Yb laser fibers with low photodarkening by optimizing of the core composition,” Opt. Express 16, 15540-15545 (2008).

[2] J. K. Sahu, S. Yoo, A. Boyland, C. Basu, M. Kalita, A. Webb, C. L. Sones, J. Nilsson, and D. N. Payne, "488 nm irradiation induced photodarkening study of Yb-doped aluminosilicate and phosphosilicate fibers," in Conference on Lasers and Electro-Optics/Quantum Electronics and Laser Science Conference and Photonic Applications Systems Technologies 2008 Technical Digest (Optical Society of America, Washington, DC, 2008), JTuA27.

[3] A. Shirakawa, K. Takaichi, H. Yagi, J-F. Bisson, J. Lu, M. Musha, and K. Ueda, "Diode pumped mode-locked $\mathrm{Yb}^{3+}: \mathrm{Y}_{2} \mathrm{O}_{3}$ ceramic laser," Opt. Express 11, 2911-2916 (2003).

[4] J. Kong, D. Y. Tang, C. C. Chan, J. Lu, K. Ueda, H. Yagi, and T. Yanagitani, "High-efficiency 1040 and 1078 nm laser emission of a $\mathrm{Yb}: \mathrm{Y}_{2} \mathrm{O}_{3}$ ceramic laser with $976 \mathrm{~nm}$ diode pumping," Opt. Lett. 32, 247-249 (2007).

[5] J. K. Sahu, M. C. Paul, M. P. Kalita, A. Boyland, C. Codemard, S. Yoo, A. Webb, R. J. Standish, J. Nilsson, S. Das, S. K. Bhadra, M. Pal, A. Dhar, and R.Sen, "Ytterbium Doped Nanostructured Optical Fibers For High Power Fiber Lasers," in European Conference on Lasers and Electro-Optics and the European Quantum Electronics Conference (Institute of Electrical and Electronics Engineers, New York, 2009), CJ2.1 WED. 